\title{
Using a digital X-ray imaging device to measure the swelling coefficient of a group of wood cells
}

\author{
E. Badel* ${ }^{*}$, P. Perré \\ LERMAB-UMR INRA 1093, ENGREF 14, rue Girardet 54042 Nancy Cedex, France
}

\begin{abstract}
An experimental device has been developed to perform numerical X-ray images by direct projection of samples made of a group of wood cells. This device consists of a micro-focus X-rays source, a scintillator and a cooled CCD camera. The dimensions are adapted to anatomical structure of wood. A specific climatic chamber has been conceived to control the moisture content of the sample. Experiments consist in imaging the sample at different humidity conditions. After pre-processing, a program compares two images and allows the bidimensional strain field to be determined. The shrinkage coefficients obtained by this protocol are representative of the unrestrained shrinkage properties of the wood sample.
\end{abstract}

Keywords: Swelling; Strain; Non-contact; X-rays; Numerical image; Wood; Image processing

\section{Introduction}

As a result of the biologic activity of the tree, wood is a material with highly varying properties both within a tree and from one tree to another. This variability is controlled by the genetic origin of the tree and by its growth conditions, from which results the anatomical structure of wood. Wood material is a very complex composite that can be studied at different observation scales. For the naked eye, wood is generally composed of layers of earlywood and latewood made up of elongated cells in the longitudinal direction of the trunk. Earlywood and latewood compose the "annual ring". Because of its very heterogeneous structure, the anatomy of oak is among the most complex in Europe. At a smaller scale, different kinds of cells are grouped and form zones that can be distinguished according to their density, shape or orientation in the transverse plane. Earlywood contains big vessels and parenchyma tissue that are produced during spring by the tree in order to conduct the rising sap to the leafs. The oak's latewood is mainly composed of parenchyma and fibre zones, which expand in summer. This part of the annual ring is very dense and is made for supporting the tree. On the other hand, big rays cells are present through the annual ring from the pith to the bark (Fig. 1).

\footnotetext{
* Corresponding author.

E-mail address: badel@engref.fr (E. Badel).
}

Different ways are conceivable to study such a complex material and its properties. We propose a determinist approach that is based on mechanical formulation. This way involves the structural characteristics of the material to be taken into consideration: the diversity of its components, their intrinsic properties and their spatial organisation are parameters that must be involved in the understanding of its physical and mechanical properties [1,2].

The difficulties of such determinist studies lie in the experimental evaluation of the wood material characteristics as:

- the quantitative evaluation of the morphological structure [3];

- the determination of the local properties.

The present work is a piece of a complete study that consists in collecting information about the structural organisation of the tissues in the annual ring and about their intrinsic properties at the microscopic level. Assuming that these data will be well known, specific mathematical formulations called "homogenisation" permit the macroscopic properties, called "homogenised", of the composite structure to be calculated. This complete study is given in detail in Refs. [4,5].

Oak is a high-value species but is well known as very difficult to be dried. Among several reasons that could explain this behaviour, shrinkage values and their anisotropy 




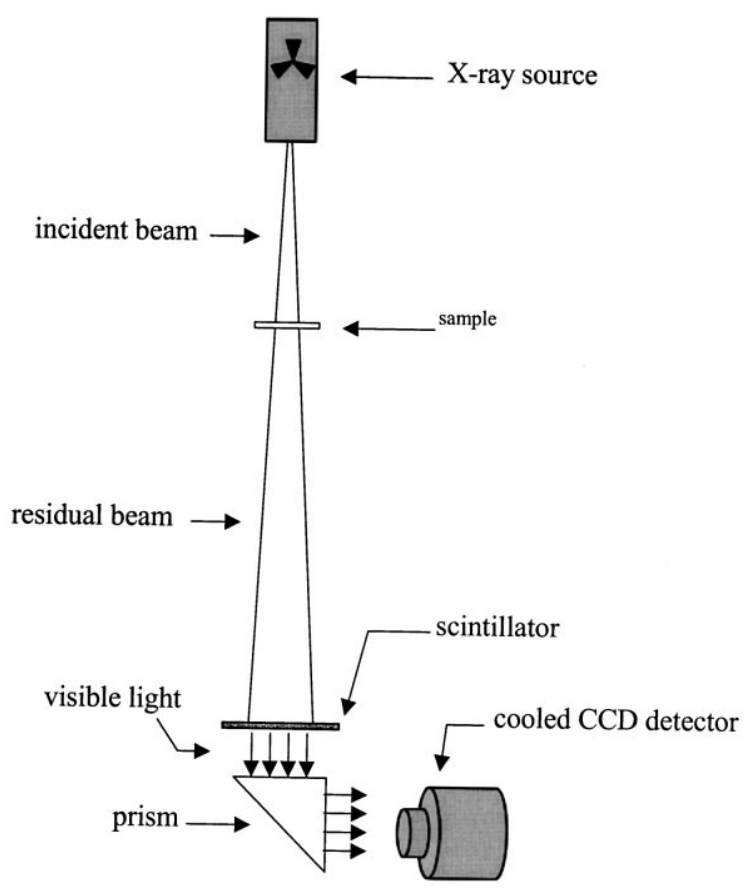

Fig. 2. Schema of the X-ray imaging device.

in the transverse plane are commonly involved. This anisotropic shrinkage in the transverse direction is considered to arise mainly from the difference in shrinkage between different components as earlywood, latewood or ray cells [3]. The modelling that results from the deterministic approach requires the microscopic shrinkage (or swelling) and mechanical properties of each tissue to be known. In this context, the evaluation of the elastic properties of very small samples is the main subject of a previous article [6].

This present paper deals with original equipment developed to measure the swelling coefficients of very small samples. The purpose of this study is to evaluate these properties for each kind of tissues (ray cells, parenchyma and fibre zones) that compose the annual ring of oak in the two directions of the transverse plane (radial and tangential).

The text is divided into three parts:

In the first part, we present the development of the experimental device, which consists of a digital X-ray imaging system equipped with an original climatic chamber.

In the second part, we propose a specific image processing developed in order to compare two images (performed at different humidity conditions) and to calculate the swelling coefficients of the sample simultaneously in two principal directions of the material.

The last part presents few experimental results. The small sizes of the wood sample (a few hundreds of micrometres) allow homogeneous tissue zones to be isolated and the shrinkage to occur without stress.

\section{Experimental device}

Any shrinkage (or swelling) experiment consists in modifying the moisture content of wood. If this variation occurs in a range from the dry state to the fibre saturation point, it results in a change of the dimensions of the sample. So, the experiment consists in following this variation according to the moisture content of the piece of wood. The first difficulty lies in the measurement of the dimensions simultaneously with the knowledge of the moisture content of the sample [7-9].

The second problem is due to the "free" shrinkage assumption. The definition of the intrinsic shrinkage properties of a piece of wood assumes that the dimensional changes due to a moisture content variation occur without external stress. The smaller is the sample, the more this hypothesis becomes important. In our case, the dimensions of the samples are less than $1 \mathrm{~mm}$ and necessitate the use of non-contact techniques of measurement. For this purpose, experimental devices use laser beam micrometers in order to measure precisely the size of the shadow of a sample along one direction. These techniques give quite good results but require the sample to have a particular shape [8]. Other systems use camera with classic lens or put on a microscope in order to follow the deformation of the surface of the sample. This one could be a thin microscopic slice [10] or a solid [8,9]. A great advantage of this method is that the information is simultaneously available in two directions.

Advances in the field of X-ray detection with the CCD offer new possibilities [11]. Especially the following of physical phenomena in time is one of these interesting possibilities. These techniques meet the two previous important criteria:

- measurement without contact;

- information in two directions.

\subsection{Design of the X-ray imaging device}

The device we developed is based on a digital X-ray imaging system that uses the principle of a direct projection of the sample on a 2D detector. The general design follows a classical schema: an X-ray source generates a polychromatic beam that is partially absorbed by the sample. The residual beam is projected onto a $2 \mathrm{D}$ scintillator. A prism deviates the visible light through the camera (Fig. 2 ). The technical specifications of the equipment are as follows:

The $X$-ray source (Hamamatsu L6731) is a micro-focus source $(\varnothing \sim 8 \mu \mathrm{m}$ at $70 \mathrm{kV})$. This material is able to work in a range of $10-80 \mathrm{kV}$ and from 0 to $100 \mu \mathrm{A}$.

The scintillator is a plate $\left(20 \times 30 \mathrm{~mm}^{2}\right)$ developed by Hamamatsu. The thin layer $(\sim 150 \mu \mathrm{m})$ of the active matter is $\mathrm{CsI}(\mathrm{Tl})$, which is presented in prismatic form. This 




Fig. 3. Structure of the fibre optic plate (FOP).

characteristic limits the diffusion of the emitted light through $\mathrm{CsI}(\mathrm{Tl})$. On the other hand, the layer is set down on a fibre optic plate $(\varnothing \sim 6 \mu \mathrm{m})$, which minimises the diffusion through the support (Fig. 3).

The camera (Princeton Instrument RTEA/CCD-1317-K) is designed for a very low noise and a low dark charge (around 15 electrons/pixel/h). In order to achieve these performances, the CCD (Kodak KAF-1400) is made of small pixels $\left(6.8 \times 6.8 \mu \mathrm{m}^{2}\right)$ and is cooled at $35^{\circ} \mathrm{C}$.

Dimensions of the device: The specific structure of wood, almost $2 \mathrm{D}$, is very appropriate for the $\mathrm{X}$-ray projection principle. However, even if the thickness of the sample can be reduced to a few hundred micrometers, the lack of sharpness resulting from the divergence of the beam leads to choose a great distance between the source and the sample. Finally we opted for a magnification equal to 3 and a source-scintillator distance equal to $175 \mathrm{~cm}$. The long acquire time resulting from this design is the most important disadvantage.

\subsection{Performances of the device}

Spatial resolution: The Contrast Transfer Function of the device is evaluated with the help of a bar/space pattern [12].

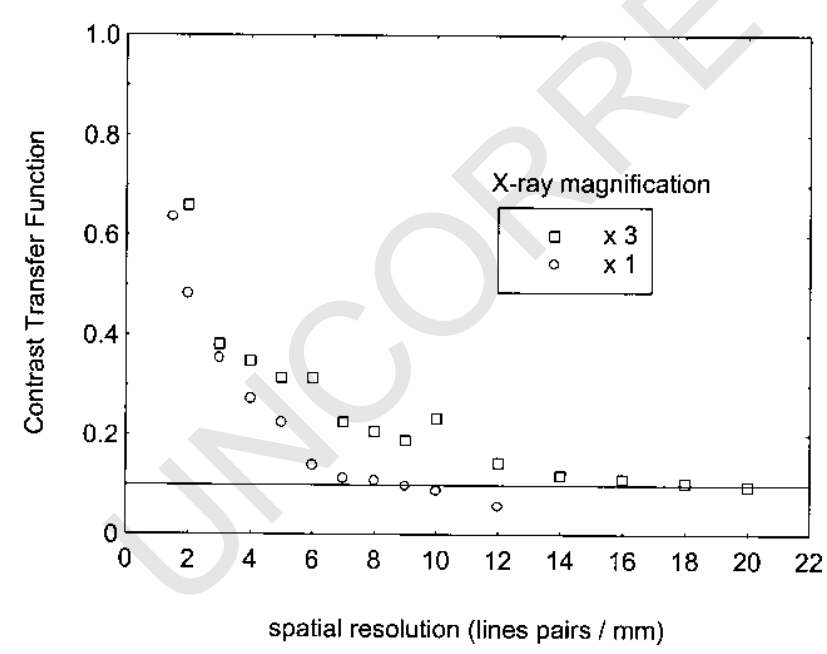

Fig. 4. Spatial resolution. The Contrast transfer function is calculated for two X-ray magnifications.
For a magnification equal to 3 , the spatial resolution of the device is around 18 lines pairs per millimetre (Fig. 4).

Quantitative resolution: Because of the better accuracy of the grey level value of each pixel with the number of photons, the sensitivity curve of the CsI material leads to use high voltage in order to increase the signal-to-noise ratio. The experimental conditions have been chosen as follows $\Delta V=$ $70 \mathrm{kV}$ and $i=95 \mu \mathrm{A}$. Fig. 5 is obtained using Kemetal ${ }^{\odot}$ blocks. The choice of this matter is motivated by its very similar atomic composition $\left(\mathrm{CH}_{2} \mathrm{O}\right)$ with cellulose $\left(\mathrm{C}_{6} \mathrm{H}_{12} \mathrm{O}_{6}\right)$, which is the most important component of wood. These experimental curves show that the great dimensions of the device and the thickness of the sample involve more than $20 \mathrm{~min}$ for a signal-to-noise ratio greater than 20 to be obtained. This long acquire time has to be compared with the kinetics of the phenomena we expect to observe.

\subsection{The climatic chamber}

In order to evaluate this characteristic time, we conducted a simple preliminary experiment. This experiment consists in following the mass loss of a totally saturated wood sample ( $\approx 1 \mathrm{mg}$ ) in room conditions with the help of an ultramicrobalance (Sartorius sc2). Fig. 6 represents the evolution of the mass: the time needed to go from the saturated state to the airdried state is only $20 \mathrm{~min}$. The comparison between the acquire time and this kinetics imposes working with static conditions. For this purpose, a small climatic chamber has been developed to stabilise the humidity rate of the air and to control the moisture content of the sample. The technical requirements for the chamber are as follows:

- to be airtight;

- to be transparent for X-rays as much as possible;

- the humidity rate of the air has to be easily modified.

The chamber is made of PVC. A central cylinder permits to position the sample on a tight-fitting membrane $\left(\right.$ mylar $\left.^{\odot}\right)$ on its top. The central section of the lid is replaced with an identical membrane and then this lid is fastened to the device, which is centred in the X-ray beam path. A container is screwed on to the device to ensure an air-tight seal. It is filled with silicate crystals or water saturated with salt. In the latter 
$20 \mathrm{kV}$

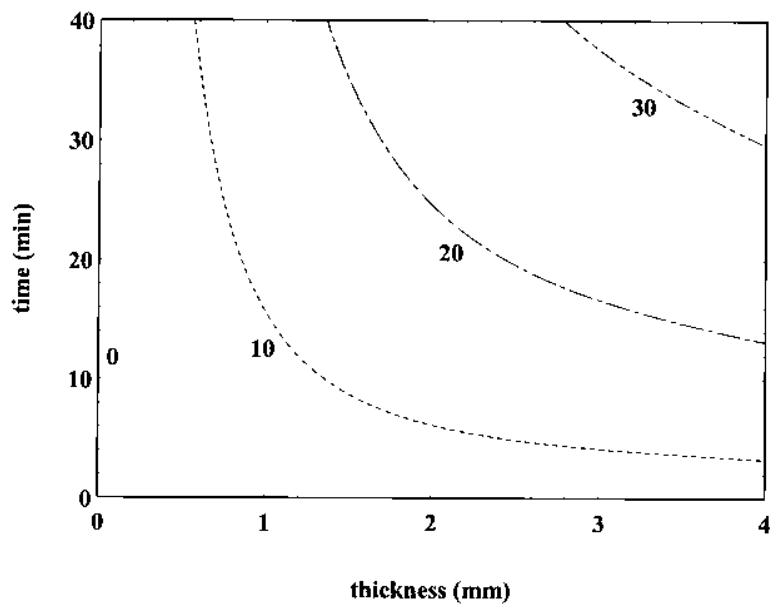

$70 \mathrm{kV}$

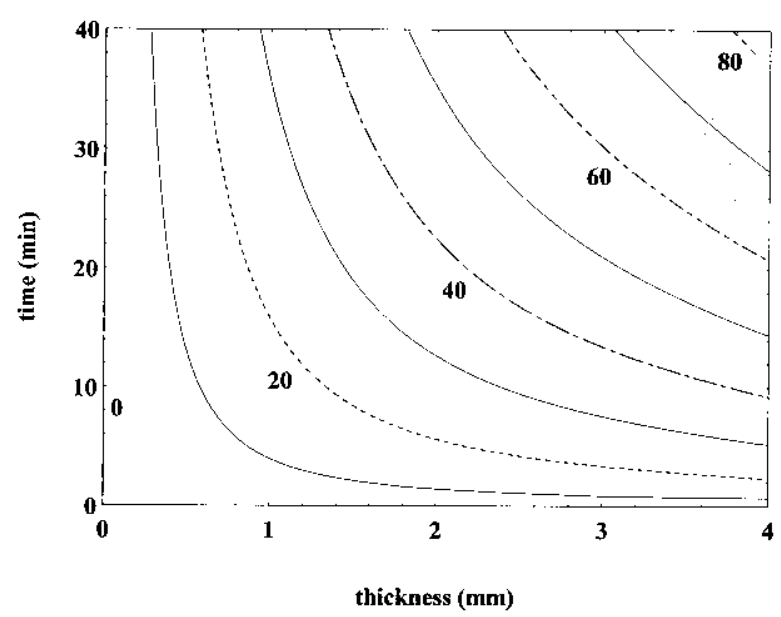

Fig. 5. Evolution of the signal-to-noise ratio according to the thickness of the sample (Kemetal ${ }^{\circledR}$ ), the acquire time and the voltage. The sensibility curve of $\mathrm{CsI}(\mathrm{Tl})$ leads to opt for higher voltage $(70 \mathrm{kV})$.

case, the choice of the nature of salt determines the humidity rate in the chamber. Because of its stability around $20^{\circ} \mathrm{C}$, we opted for a $\mathrm{NaCl}$ solution. Two sensors measure the temperature and the relative humidity of air (Fig. 7) that are recorded. These data are essential for the estimation of the moisture content of the sample.

\subsection{Sampling and experiment}

In Section 1, we saw that the only possible way to measure the unrestrained shrinkage of a tissue is to isolate it. At first, a thin slide (thickness $\approx 400 \mu \mathrm{m}$ ) is cut in the transverse plane with the help of a micro-circular saw. Then, the other sides of the sample are sliced with a razor blade (Fig. 8). At this step, the sample is fully soaked in water when it is placed in the climatic chamber. In this first phase, the solution of $\mathrm{NaCl}$ produces a high humidity rate $(\approx 70-$
75\%). After stabilisation of the conditions, a first image is recorded. Then the solution is replaced by silicate crystals. After air-drying, the moisture content of the sample decreases by $\approx 10 \%$. After this new stabilisation, a second image of the image is recorded. In both cases, the moisture content of the sample is evaluated according to classical curves, well known in wood science, called "sorption isotherms". During the first step (solution of $\mathrm{NaCl}$ ), the moisture content of the sample $(\mathrm{MC}=$ mass of water/dry mass) is around $13 \%$ whereas this value decreases until about $3 \%$ with the use of silicate crystals.

\section{Measurement of the swelling coefficients}

\subsection{Image pre-processing}

A swelling coefficient of wood is defined as the deformation



Fig. 6. Test of drying speed of a small fibre sample (room conditions). 

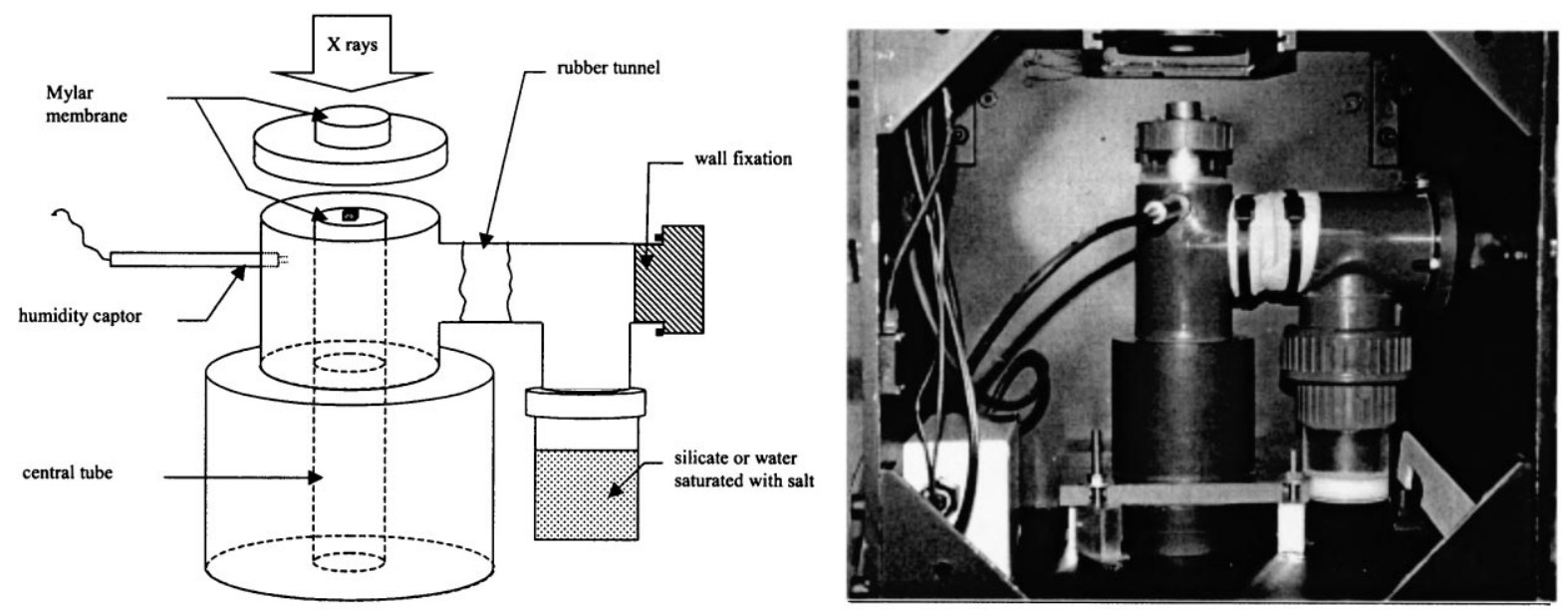

Fig. 7. Climatic chamber integrated in the X-ray device. A first solution of water and salt produces a high humidity rate. Then it is replaced by silicate that dries the air.

induced by a variation of $1 \%$ of the moisture content. So, its calculation requires the strain field due to the moisture content change to be known. This one is evaluated by comparison of the two experimental images [13]. This specific process required a first procedure in order to isolate the sample and to get its shape.

Assuming that the dimensions of the sample on the image will highly depend on this operation, the choice of the threshold limit on the grey levels histogram has to be an automatic procedure. The method we use is based on the modelling of each group of grey levels (here, only two) as a Gaussian distribution (Fig. 9). The Fisher's method [14] consists in finding the separator that minimises the inertia of the two groups:

$I=\sum_{g \in \mathrm{gr}_{1}} W(g)\left(g-c\left(\mathrm{gr}_{1}\right)\right)^{2}+\sum_{g \in \mathrm{gr}_{2}} W(g)\left(g-c\left(\mathrm{gr}_{2}\right)\right)^{2}$

where $c$ is inertia centre of the group, $g$ the grey level and $W$ the weight of the grey level (number of pixel having the value " $g$ "). $\mathrm{gr}_{1}$ and $\mathrm{gr}_{2}$ are the two groups corresponding to the sample and the background.

Using this threshold value, a classic image processing (composed of "erosion", "reconstruction" and the "hole fill" operations) permits the sample to be correctly isolated.

\subsection{Determination of the strain field}

The main principle consists in distorting the image no. 1 by applying a virtual strain field. The resulting calculated image is compared with the experimental image no. 2. A specific program determines the strain field, which permits the calculated and the second experimental image (no. 2) to be as identical as possible (Fig. 10).

The virtual image is computed as follows. Consider two points $(A$ and $B$ ) of a solid. After deformation and displacement, the new position of the point $B$ is:

$\overrightarrow{O B} \cdot=\overrightarrow{O A}+\overrightarrow{A A} \cdot \overrightarrow{A^{\prime} B^{\prime}}$

with

$\overrightarrow{A^{\prime} B^{\prime}}=(\overline{\bar{R}} \leftrightarrow \overline{\bar{S}}) \overrightarrow{A B}$

$\overrightarrow{A A^{\prime}}$ is the solid translation in the $x y$-plane (two parameters), $\overline{\bar{R}}$ is the rotation tensor (anti-symmetric; one parameter) and $\bar{S}$ is the strain field tensor (symmetric; three parameters):

$\overline{\bar{R}}=\left(\begin{array}{cc}\cos \theta & -\sin \theta \\ \sin \theta & \cos \theta\end{array}\right) \quad\left(\begin{array}{ll}\varepsilon_{x x} & \varepsilon_{x y} \\ \varepsilon_{x y} & \varepsilon_{y y}\end{array}\right)$

where $\varepsilon_{x x}$ is the strain in the $x$-direction, $\varepsilon_{y y}$ the strain in the $y$-direction, $\varepsilon_{x y}$ the shear strain and $\theta$ the angle of rotation.

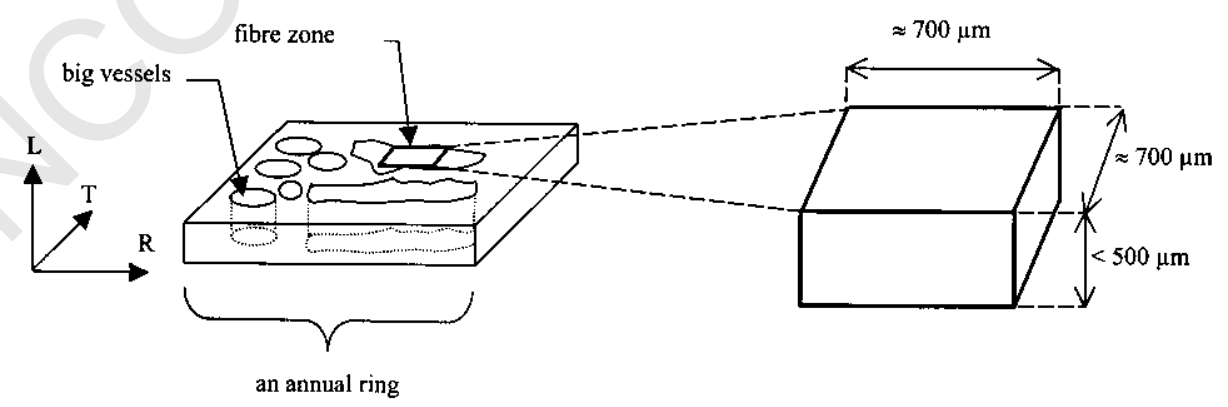

Fig. 8. Sample preparation: a thin slide is cut in the longitudinal direction and then the tissue is isolated. 


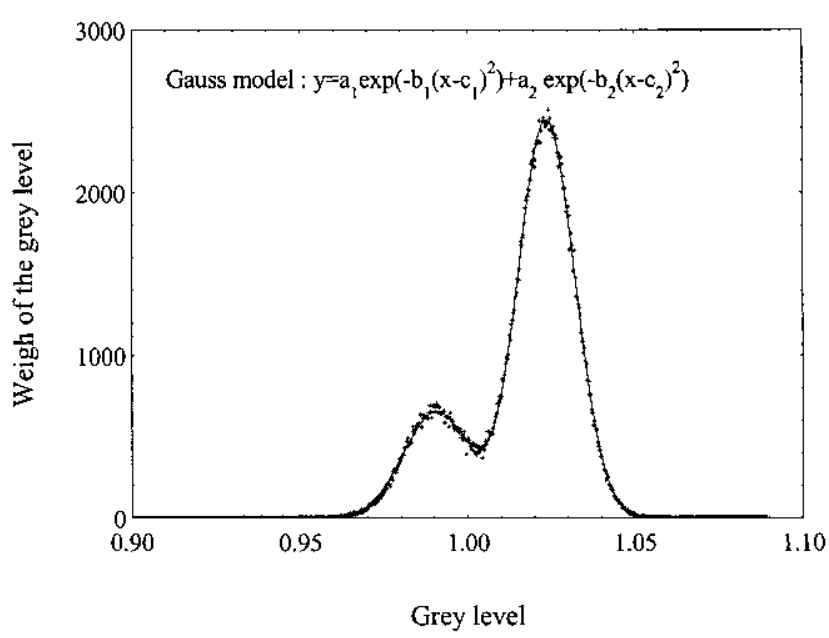

Fig. 9. Grey levels histogram. Each group (background and sample) is modelled as a Gaussian distribution.

Finally, six parameters are required for the calculation of the virtual image. The comparison between the virtual deformed image and the real deformed image (no. 2) involves their grey level distribution. The difference between theses two distributions can be quantified by a criterion $C$ :

$C=\frac{\sum_{\text {image }}\left(G_{\text {virtual }}-G_{\text {exp }}\right)^{2}}{N}$

where $G_{\text {virtual }}$ (resp. $G_{\text {exp }}$ ) is the grey level of pixel on the calculated image (resp. on the actual image) and $N$ the number of pixels in the image. The minimisation of this criterion permits the six parameters (three for the solid

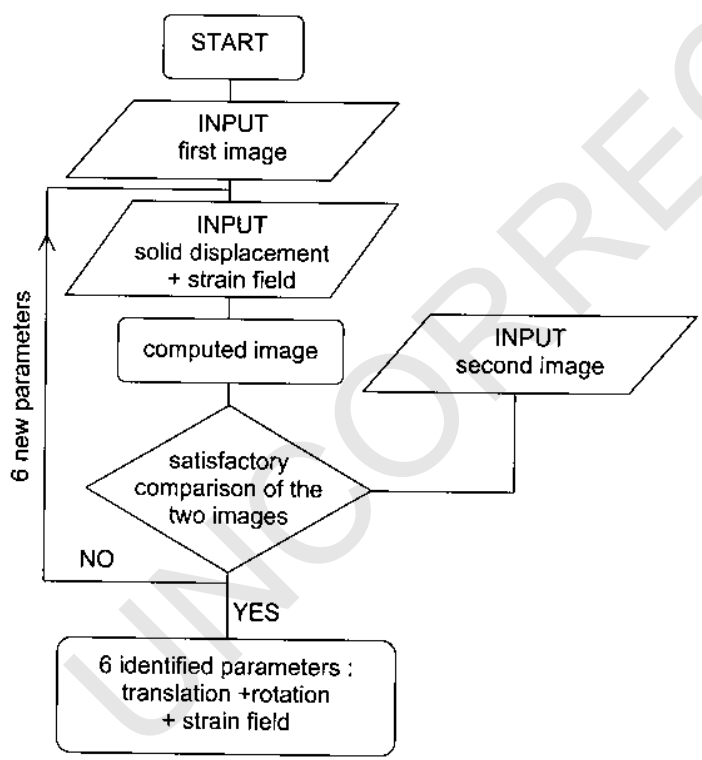

Fig. 10. Strain field determination. The comparison of the grey level distributions on the two images permits the solid displacements and the strain field to be determined. displacement + three for the strain field) to be estimated. They correspond to the distortion of the image. In particular, the values of $\varepsilon_{x x}, \varepsilon_{y y}$ and $\varepsilon_{x y}$ are calculated in the two physical directions ( $x$ and $y$ ) of the image. The calculation of strain in the two principal directions of the material (radial and tangential) is obtained by rotation of the tensor $\overline{\bar{\varepsilon}}$. Assuming that free shrinkage occurs without stress, the resulting deformation does not involve shear $\left(\varepsilon_{x y}=0\right)$. In this new orthogonal system, the tensor $\overline{\bar{\varepsilon}}$ becomes diagonal. Finally, the strain values $\lambda_{1}$ and $\lambda_{2}$ in the principal directions of the material are the solutions of the eigenvalue problem:

$[\overline{\bar{\varepsilon}}-\lambda \overline{\bar{I}}]=0$

where $\overline{\bar{I}}$ is the identity tensor.

\subsection{Calculation of swelling coefficients}

In the hygroscopic domain between the dry state and the fibre saturation point, the strain is proportional to the moisture content. This permits the swelling coefficient to be calculated as the ratio:

$\alpha=\frac{\Delta \varepsilon}{\Delta H} \quad$ with $\varepsilon_{H}=\frac{l_{H}-l_{0}}{l_{0}}$

where $\varepsilon_{H}$ is the strain (calculated using the dry state as reference), $H$ the moisture content and $l_{H}$ the dimension of the sample $\left(l_{0}\right.$ for $\left.H=0 \%\right)$.

The data we obtained by image comparison correspond to the strain field $\varepsilon_{\mathrm{m}}$ of the sample between two states $\left(H_{1}\right.$ and $\mathrm{H}_{2}$ ) and is defined as follows:

$\varepsilon_{\mathrm{m}}=\frac{l_{H_{2}}-l_{H_{1}}}{l_{H_{1}}}$

where $\epsilon_{\mathrm{m}}$ is the measured strain field by image comparison and $l_{H_{1}}$ and $l_{H_{2}}$ are the dimensions of the sample.

Assuming that the definition of the strain involves the dry state $(H=0 \%)$ as initial configuration, the swelling coefficient can finally be calculated according to the Eqs. (4) and (5) as follows:

$$
l_{H}=l_{0}(1+\alpha H) \quad \Rightarrow \alpha=\frac{\varepsilon_{\mathrm{m}}}{\left(H_{2}-H_{1}\right)-H_{1} \varepsilon_{\mathrm{m}}}
$$

\section{Few results of swelling experiments}

The easy procedure to isolate a piece of tissue, which permits to choose samples very close in the same part of a tree (Fig. 11). This possibility limits the problems of variability between two trees and/or two positions in the trunk, which is well known to make interpretations of the results difficult in wood science [15].

The duration of each experiment is around 2 days. Keeping in mind that the main objective of this study is to obtain the rough estimate of the swelling values for the 




Fig. 11. Sampling. A piece of fibre is isolated from the annual ring.

components oak's anatomy, the number of samples has been limited. We performed fives experiments with ray cells, four with fibre zone and four with parenchyma zones. Fig. 12 shows the experimental results. The values of the swelling coefficients are expressed in strain (\%) per moisture content variation (expressed in \%) and are calculated according to the Eq. (6).

These original data show that the tissues of oak have very different swelling behaviours. Ray cells have very low coefficient in the radial direction. This result corroborates the findings in the literature: because of the specific orientation of their cells in the radial direction, ray cells are commonly involved in restraining the shrinkage during the drying in this direction [16,17]. On the other hand, the variability of the experimental result in the tangential direction does not allow us to reach this conclusion. The specific elongated aspect of the samples could explain this variability of the results. Few tests conducted with drawn rectangles seem to show that the image comparison process would be less efficient in such particular cases.

Fibre zones present the strongest swelling coefficients. In tangential direction, such values $(0.58 \% / \%)$ would provide a size variation of around $11 \%$ during a modification of the moisture content of $20 \%$ in the hygroscopic domain (for example, from the fully soaked state to the air-dried state). This result is in accordance with studies, which observed a peak of the swelling values in case of important proportion of fibre in the annual ring of oak [7]. On the other hand, fibre tissues reveal a great anisotropic behaviour. With lower values, the same observation can be noticed for the parenchyma zone. In both cases, the tangential to radial ratio is around two.

\section{Conclusions}

This paper presented an experimental method for the measurement of the swelling properties of very small wood sample. Using X-ray images as a source of information, the main characteristic of this experiment is the noninterference between the strain and its measurement that makes sure that the sample to be unrestrained. The principle of images comparison, initially developed for mechanical tests, permitted to measure strain due to moisture content changes simultaneously in two directions. The climatic chamber permitted the moisture content of the wood sample to be stabilised.

In this work, exposure conditions have been chosen in order to separate easily the sample from the background. In a next step, we study the possibility to adapt them in order to evaluate the moisture content of the sample according to the X-ray attenuation variations. This direct measurement assumes a great precision of the signal.

The results show that the tissues of oak have different intrinsic properties. Fibre zones present the strongest values and ray cells the lowest. If the wood anisotropy is commonly considered to arise mainly from the interaction between the different components of the annual ring, these

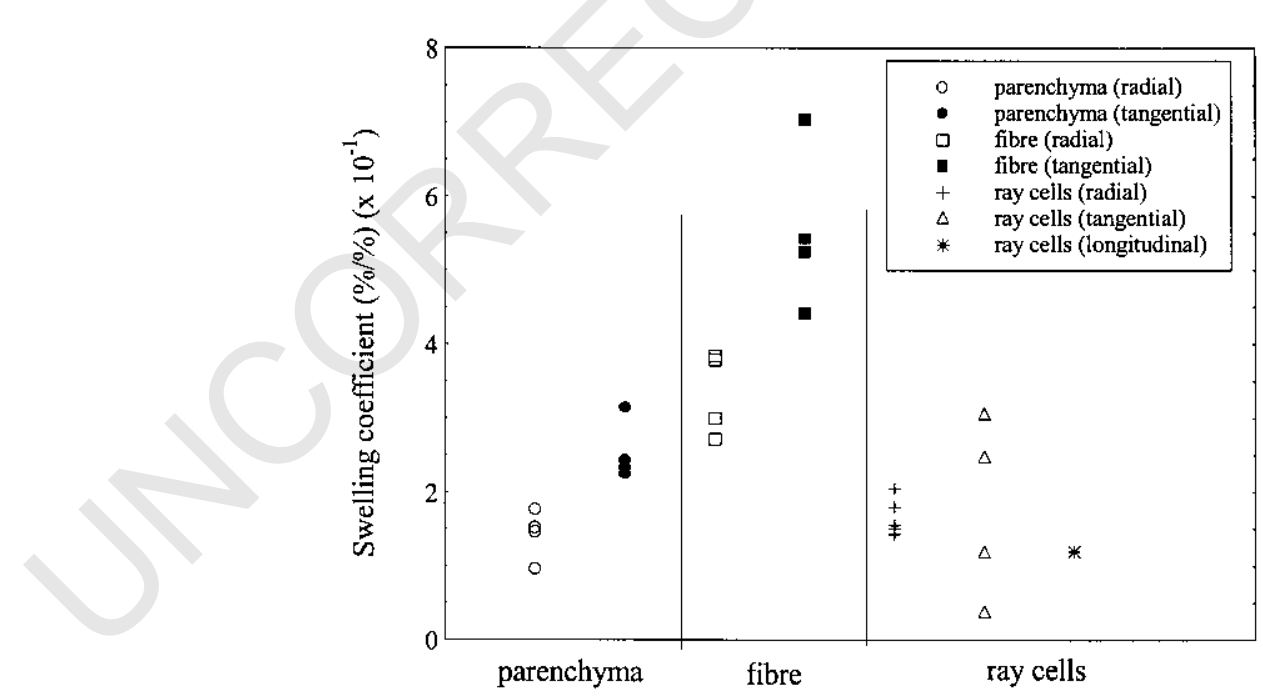

Fig. 12. Results: experiments have been performed especially in the radial and the tangential direction of the trunk. Swelling coefficients are calculated according to Eq. (6) and correspond to the strain value for a $1 \%$ variation of the moisture content of the sample. 
results also show anisotropy to be an intrinsic behaviour of each tissue.

These data are essential to model the swelling behaviour of oak. This work consists of two steps. First the spatial distribution of the tissues inside the annual ring is collected using the X-ray imaging device. Then, finite elements mesh, which represents this structure is built and homogenisation techniques permits the macroscopic properties of the complete annual ring to be computed.

\section{References}

[1] Kollman FFP, Cote WA. Principles of wood science and technology. Berlin: Springer, 1984.

[2] Gibson LJ, Ashby MF. Cellular solids. Oxford: Pergamon Press, 1988.

[3] Watanabe U, Fujita M, Norimoto M. Transverse shrinkage of coniferous wood cells examined using replica method and power spectrum analysis. Holzforshung 1998;52(2):200-6.

[4] Badel E. Détermination des propriétés élastiques et du retrait d'un cerne annuel de chêne dans le plan transverse: description de la morphologie, mesures des propriétés microscopiques et calculs d'homogénéisation. Thesis. ENGREF — Nancy, 1999. 178 pp.

[5] Badel E, Perré P. The modelling of elastic and shrinkage properties of wood in transverse directions using homogenisation techniques. 3rd Plant biomechanics Conference, Freiburg, 2000. p. 391-6.

[6] Badel E, Perre P. Determination des proprietes elastiques d'elements individuels du plan ligneux du chene par des essais de traction sur micro-eprouvettes. Ann For Sci 1999;56:467-78.

[7] Botosso P. Une méthode de mesure du retrait microscopique du bois:
Application à la prédiction du retrait tangentiel d'éprouvettes de bois massif de sapin pectiné (Abies alba Mill.). Thesis. University of Nancy I, 1997.

[8] Mariaux A, Masserann C. Anisotropie de retrait et structure du bois. Recherche de l'influence des caractère morphologiques transverse des fibres. Bois et Forets de Tropiques 1985;209:35-47.

[9] Lahbabi R. Mesure par analyse d'image de parametres microscopiques requis pour une prediction deterministe des proprietes du bois. Thesis. ENGREF — Nancy, 1995.

[10] Boutelje J. On shrinkage and change in microscopic void volume during drying, as calculated from measurements on microtome cross sections of Swedish pine. Holzforshung 1962;65:209-15.

[11] Rondot S, Cazaux J. Microscopie X analytique et diffusion des ions en solution. Colloque C4, Supplement au J Physique III 1996;6:733-8.

[12] Kaftandjian V, et al. A comparison of the ball, wire, edge and bar/ space pattern techniques for modulation transfer function measurements of linear X-ray detectors. J X-ray Sci Technol 1996;6:205-21.

[13] Farruggia F, Perre P. Microscopic tensile tests in the transverse plane of earlywood and latewood parts of spruce. Wood Sci Technol 2000;34:62-82.

[14] Coquerez JP, Philipp S. Analyse d'images: filtrage et segmentation. Paris: Masson Press, 1998.

[15] Eyono Owondi R. Modelisation de la retractabilite du bois en relation avec des parametres de la structure de l'accroissement annuel et de la position dasn l'arbre chez Quercus Robur L. et Q. Petraea Liebl. Thesis. ENGREF — Nancy; 1992.

[16] Kawamura Y. Studies on the properties of rays. III. Influence of rays on anisotropic shrinkage of wood. Mokuzai Gakkaaishi 1984;30(10):785-90.

[17] Wijesinghe S. The shrinkage of rays and fiber in wood. Forestry 1959;32(1):31-38. 